INTERNATIONAL JOURNAL OF

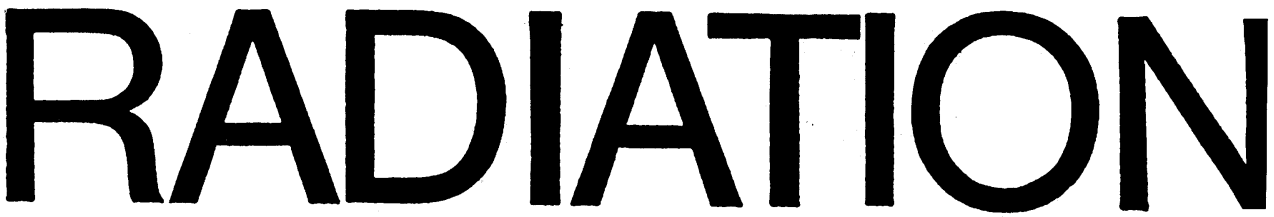

Volume 57

Number 4

April

1990
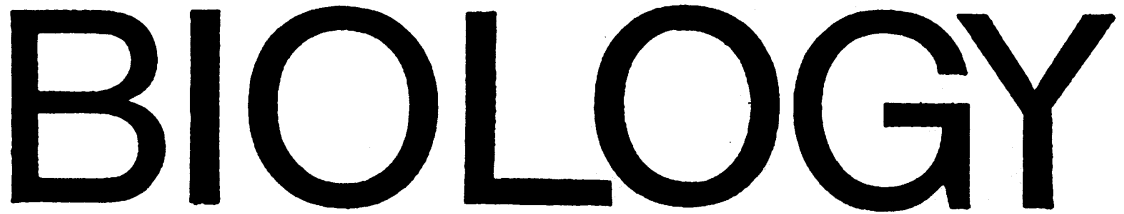

Covering the physical, chemical, biological, and medical effects of ionizing and non-ionizing radiations

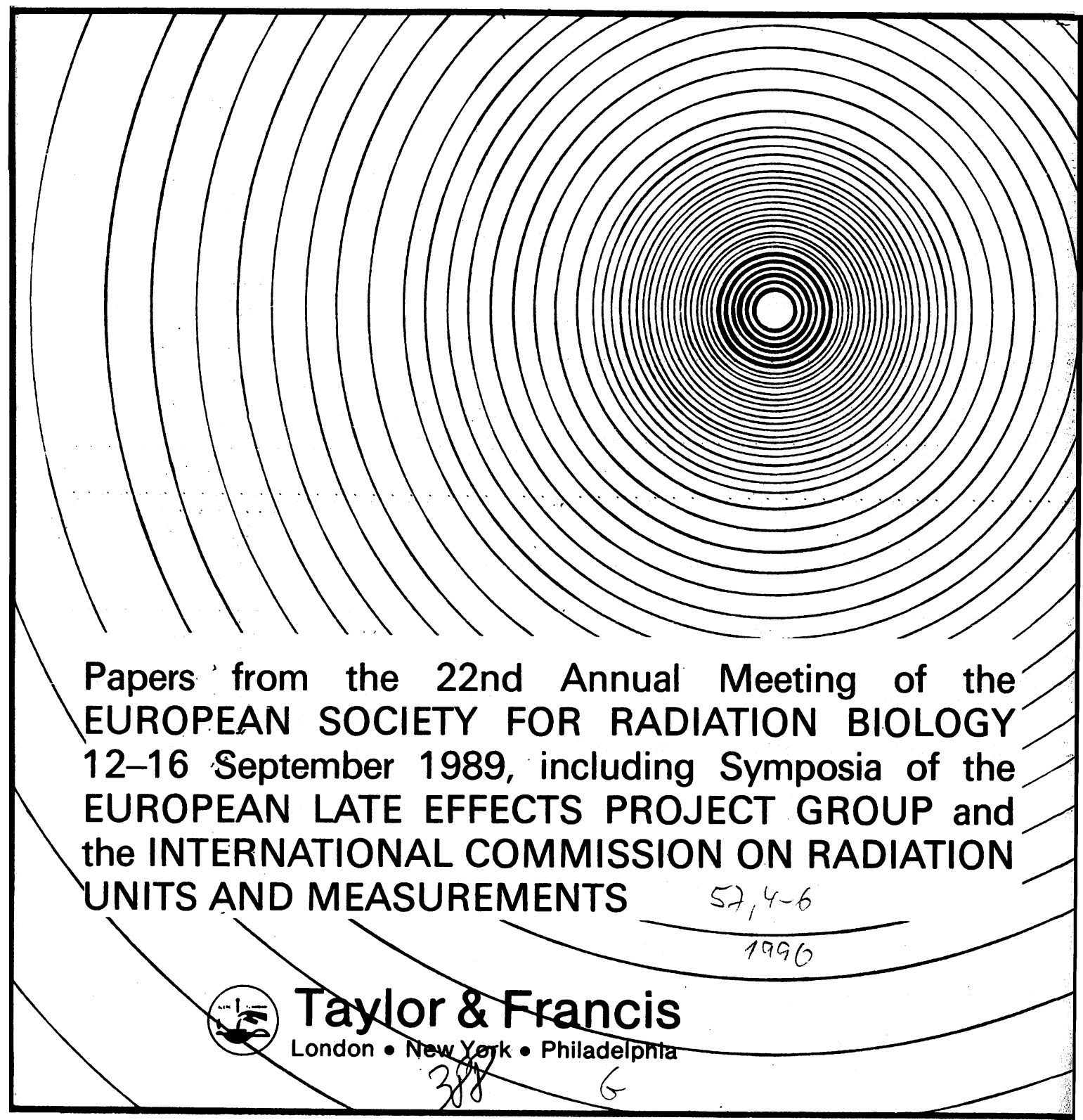




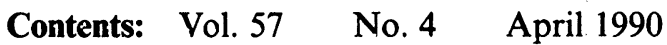

\section{2nd ANNUAL MEETING OF THE EUROPEAN SOCIETY FOR RADIATION BIOLOGY}

\section{POPULATION EXPOSURE AND EFFECTS}

Environmental radiation and radioactive releases. A. Aarkrog. . $\quad \ldots \quad \ldots \quad \ldots$

Dosimetric aspects of exposure of the population to ionizing radiation. J. J. Broerse

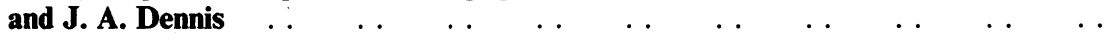

The effect of prenatal radiation exposure on the developing human brain. R. H. Mole

\section{MUTAGENESIS AND CARCINOGENESIS}

Mechanisms and consequences of mutation induction in mammalian cells. A. Sarasin, F. Bourre, L. Daya-Grosjean, A. Gentil, C. Madzak and A. Stary .

The molecular biology of radiation-induced carcinogenesis: thymic lymphoma, myeloid leukaemia and osteosarcoma. M. Janowski, R. Cox and P. G. Strauss

Cellular events in radiation-induced lymphomagenesis. J. Boniver, C. Humblet, A. M. Rongy, C. Delvenne, P. Delvenne, R. Greimers, A. Thiry, R. Courtoy and M. P.

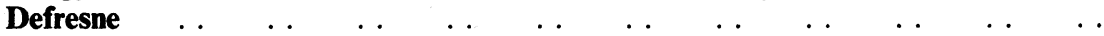

\section{PROTECTION}

Trends and developments in radioprotection: the effect of nicotinamide on DNA repair. E. Riklis, R. Kol and R. Marko . $\quad \ldots \quad \ldots \quad \ldots \quad \ldots \quad \ldots \quad \ldots$

Advances in radioprotection through the use of combined agent regimens. J. F. Weiss, K. S. Kumar, T. L. Walden, R. Neta, M. R. Landauer and E. P. Clark . .

Therapeutic use of recombinant human G-CSF (rhG-CSF) in a canine model of sublethal and lethal whole-body irradiation. T. J. MacVittie, R. L. Monroy, M. L. Patchen and L. M. Souza . . . . . . . . . .

EULEP SYMPOSIUM: Skin-its relevance in radiation accidents and radiological protection

DOSIMETRY, RESPONSES AND TREATMENTS

Dosimetry for radiation accidents: present status and prospects for biological

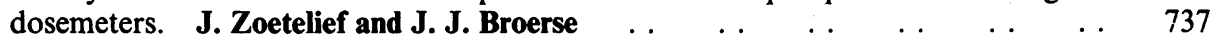

The skin: its structure and response to ionizing radiation. J. W. Hopewell . . . $\quad 751$

The dependence of skin lesions on the depth-dose distribution from $\beta$-irradiation of people in the Chernobyl nuclear power plant accident. A. Barabanova and D. P. Osanov.

. . $\quad \ldots \quad \ldots \quad \ldots 775$

Medical and surgical management for localized radiation injuries. J. C. Nénot $\quad \ldots \quad 783$

CARCINOGENESIS AND RISKS

Experimental studies of radiation carcinogenesis in the skin: a review. J. E. Coggle

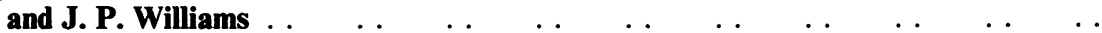

Overview of radiation-induced skin cancer in humans. $\quad$ R. E. Shore $\quad \ldots \quad \ldots \quad \ldots \quad \ldots 09$

Radiation protection guidelines for the skin. $\quad$ R. J. M. Fry $\quad \ldots \quad$. $\quad \begin{array}{llllll} & \ldots & \ldots & 829\end{array}$

General considerations of the choice of dose limits, averaging areas and weighting factors for the skin in the light of revised skin cancer risk figures and experimental data on non-stochastic effects. M. W. Charles . 
ICRU SYMPOSIUM: Energy deposition, specification of radiation quality, and the shape of the dose-response curve in radiation protection and therapy

A generalized definition of dosimetric quantities. A. M. Kellerer and H. H. Rossi . .

Microdosimetric specification of radiation quality in neutron radiation therapy H. G. Menzel, P. Pihet and A. Wambersie $\quad \ldots \quad \ldots \quad \ldots \quad \ldots \quad \ldots \quad$. .

Mechanisms of cell reproductive death and shapes of radiation dose-survival curves of

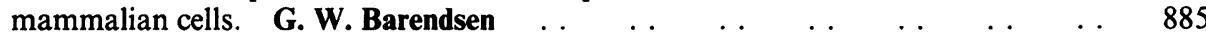

$\begin{array}{lllllllllllll}\text { Diary of events } & \ldots & \ldots & \ldots & \ldots & \ldots & \ldots & \ldots & \ldots & \ldots & \ldots & \ldots & 897\end{array}$

$\begin{array}{llllllllllll}\text { Forthcoming papers } & \ldots & \ldots & \ldots & \ldots & \ldots & \ldots & \ldots & \ldots & \ldots & \ldots & 902\end{array}$

Copyright (C) 1990 Taylor \& Francis Ltd

All rights reserved. Authors are themselves responsible for obtaining permission to reproduce copyright material from other sources and are required to sign a form for agreement of the transfer of copyright. All requests from third parties to reprint material held in copyright by Taylor \& Francis must be referred to the author for consent as a condition of the granting by Taylor $\&$ Francis of permission for reproduction. No part of this publication may be reproduced, stored in a retrieval system or transmitted in any form, or by any means, electronic, electrostatic, magnetic, mechanical, photocopying, recording or otherwise, without prior permission in writing from the copyright holder.

Authorization to photocopy items for internal or personal use, or the internal or personal use of specific clients, is granted by Taylor \& Francis Ltd for libraries and other users registered with the Copyright Clearance Center (CCC) Transactional Reporting Service, provided that the base fee is paid directly to CCC, 27 Congress Street, Salem, Massachusetts 01970, USA. (For fee per article, see copyright code on first page of each article.) This consent does not extend to other kinds of copying, such as copying for general distribution, for advertising, for creating new collective works, or for resale. Fees for past articles are the same as those shown for current articles. 
INT. J. RADIAT. BIOL., 1990, vOL. 57, NO. 4, 859-864

\title{
A generalized definition of dosimetric quantities*
}

\author{
A. M. KELLERER $\dagger \ddagger$ and H. H. ROSSI§ \\ $\dagger$ Institut für Medizinische Strahlenkunde der Universität Würzburg, \\ Versbacher Strasse 5, D-8700 Würzburg, FRG \\ $\S 105$ Larchdale Ave., Upper Nyack, NY 10960, USA
}

The current definitions of microdosimetric and dosimetric quantities use the notion of 'ionizing radiation'. However, this notion is not rigorously defined, and its definition would require the somewhat arbitrary choice of specified energy cut-off values for different types of particles. Instead of choosing fixed cut-off values one can extend the system of definitions by admitting the free selection of a category of types and energies of particles that are taken to be part of the field. In this way one extends the system of dosimetric quantities. Kerma and absorbed dose appear then as special cases of a more general dosimetric quantity, and an analogue to kerma can be obtained for charged particle fields; it is termed cema. A modification that is suitable for electron fields is termed reduced cema.

\section{Introduction}

The International Commission on Radiation Units and Measurements (ICRU) has always seen it as a central part of its responsibilities to extend the quantitative tools of radiation dosimetry and radiation biology. The historical transition from the earlier radiation units and quantities to exposure and then to absorbed dose, and the introduction of the International System of Units into radiation dosimetry attest to this aim. 'The formulation of concepts that specify the quality of a radiation with regard to the microscopic and macroscopic spatial patterns of energy deposition has been a further aspect of particular importance to radiation biology and radiation protection. In the work of ICRU it is reflected by the formal introduction of the concepts of microdosimetry into the system of basic radiation quantities (ICRU 1980,1983 ); and by the recent definition of the new radiation protection quantities, such as ambient dose-equivalent or directional dose-equivalent (ICRU 1985, 1988).

The focus of this symposium is on the microdosimetric quantities and their use in radiation biology, radiology and radiation protection. Microdosimetry originated about 30 years ago, but it is still in a state of development and expansion that seems to set it apart from the classical areas of dosimetry where few, if any, changes of basic quantities are still expected. The introduction of the 'stochastic' radiation quantities may, however, not be the last major change in the conceptual framework of dosimetry. 'This initial contribution to the symposium deals with a basic defect at the very outset of the system of definitions of ICRU that leads from the individual energy exchanges in particle interactions to the inchoate energy distribution, to energy imparted, to specific energy, and finally to the various conventional

* Presented at the International Commission on Radiation Units and Measurements (ICRU) Symposium at the 22nd Annual Meeting of the European Society for Radiation Biology, 12-16 September 1989, Brussels.

$\ddagger$ Author for correspondence. 
dosimetric quantities. It will be seen that the definition of the concept of ionizing radiation lacks rigour; but it will also be recognized that this lack of rigour is a necessary consequence of the fact that the current set of microdosimetric and dosimetric quantities is merely a subset of a broader system of quantities. A brief consideration of the generalized definitions will lead to certain useful intermediate dosimetric quantities, but the generalized formulation will also serve the second major responsibility of ICRU, which is the constant attempt at unification that needs to accompany the emergence of new or modified quantities.

\section{A problem in the definition of ionizing radiation}

The joint basis of all microdosimetric and dosimetric quantities is the notion of ionizing radiation. This notion is seemingly simple; ionizing radiation is stated to consist of uncharged or charged particles capable of ionizing matter (ICRU 1980). On closer examination one recognizes the deficiencies of this definition. Whether a radiation is ionizing or not depends on the exposed material, and any actual definition therefore requires an arbitrary choice. The difficulty goes deeper; one can choose a material, e.g. biological tissue, but there is no rigorous definition of the process of ionization in a condensed material. An actual substrate contains at least some shallow energy levels that permit ionization even by low-energy electrons; there is thus no defined lower-energy limit for the process.

One concludes that 'ionization' is not a suitable notion for the definition of 'ionizing radiation'. A pragmatic definition requires, instead, a chosen cut-off energy for a given type of particle. Such a convention remains arbitrary, but it is at least well defined.

The current system of definitions by ICRU implies the need to choose a cut-off energy, but does not make a choice. The resulting uncertainty is a matter of some practical concern, since it can lead to substantial differences in microdosimetric computations with charged particle simulation codes. However, it is more significant as an indication that the system of basic definitions ought to be generalized. Instead of choosing one specific cut-off energy-e.g. within the range $2-15 \mathrm{eV}$ for electrons-one can do the opposite and consider the modified system of definitions that results from an entirely free choice of cut-off energies for different types of particles. In effect this amounts to replacing the notion of a field of ionizing radiation by that of 'reduced' fields that include only a stated category, $K$, of particles and particle energies. The resulting generalizations of quantities such as energy deposit, energy imparted, specific energy, or finally absorbed dose will not be given here; the reader is instead referred to a more detailed account (Kellerer and Rossi, manuscript in preparation). But the general concept will be exemplified by the subsequent consideration of kerma and of analogous intermediate dosimetric quantities.

\section{Kerma as an intermediate dosimetric quantity}

The interaction of ionizing radiation with matter can be seen as a series of successive random steps of energy degradation. Neglecting certain pathways that are often of minor importance one can, for example, say that fast neutrons degrade their kinetic energy by converting it stepwise into kinetic energy of charged recoils. The charged recoils, in turn, convert in successive interactions part of their kinetic energy into kinetic energy of released delta rays and expend another part against binding energy in the exposed material. The distribution of absorbed dose in the 
exposed material is determined by all steps of this degradation process. But in many computations, and even in measurements, energy transport by the charged particles can be disregarded. In the sense of the generalized definitions this corresponds to accounting only for the category of uncharged particles, and instead of absorbed dose one then obtains a quantity that is nearly equal to kerma. It differs from kerma merely in including also the minor fraction of energy expended by the uncharged particles against binding energy, i.e. the fraction that does not appear as kinetic energy of the released charged secondaries.

The definition of kerma (Roesch 1958, ICRU 1980) reflects the fact that energy transport by uncharged particles is often mainly responsible for the spatial patterns of energy imparted, while the energy dissipation by charged secondaries can be neglected. Kerma is a suitable intermediate dosimetric quantity under this condition. It facilitates calculations, but, perhaps more importantly, it indicates, for a radiation free in air or even in vacuum, a value of 'dose' that comes close to actual absorbed doses in potential receptors without being dependent on the particularities of a specific receptor. Its most essential role is therefore that of a reference quantity for calibration purposes which is, unlike the specialized radiation protection quantities dose (equivalent) index, ambient dose (equivalent), or the directional dose (equivalent), independent of a selected receptor geometry.

The existence and the applicability of kerma pose the obvious question of whether one can define an analogous intermediate dosimetric quantity for fields of charged ionizing particles.

\section{Cema}

A quantity that is largely analogous to kerma can be readily defined if one deals with charged particles other than electrons; the term 'ions', will be used in the following, although such radiations as mesons or positrons are also admitted. For a radiation field of ions one can consider the integral over the charged particle fluence spectrum in energy, $\varphi(T)$, times the stopping power, $I,(T)$ :

$$
C=\frac{1}{\rho} \int_{0}^{\infty} \varphi(T) L(T) \mathrm{d} T
$$

where $\rho$ is the density of the material and $\varphi(T) \mathrm{d} T$ is the fluence of particles with energy between $T$ and $T+\mathrm{d} T$.

We will call this quantity cema (converted energy per unit mass). In the sense of the generalized definitions it results instead of absorbed dose when one considers the category of all charged particles except delta rays. Where kerma disregards all energy transport by the charged particles, cema disregards merely the energy transport by delta rays. If one deals with high-energy radiation in space, the range of the delta rays can be large, and cema can then substantially differ in its spatial distribution from absorbed dose. In general, however, cema will differ less from absorbed dose than does kerma. This is so because the delta-ray ranges are usually short. A further characteristic difference is that charged particles-unlike photons or neutrons-expend a considerable part of their kinetic energy directly against binding energy in the material.

In the present context only basic aspects of the intermediate dosimetric quantities are discussed, and the definitions are related merely to non-stochastic variables. However, it will be evident that similar considerations apply to the associated stochastic quantities. A microdosimetric analogue of cema has, indeed, 
been used in most microdosimetric computations for neutrons; in such calculations energy transport by the heavy recoils is taken into account, but the energy transport by their delta rays is usually disregarded (Caswell and Coyne, 1972). The concept of cema can be helpful for a better classification of this and of similar approximations.

\section{The difficulty of recursive energy conversion}

The cascade of energy conversions can for a radiation of uncharged particles or of ions be conveniently subdivided into consecutive steps, but its description becomes complicated if one includes recursive processes such as the bremsstrahlung by charged particles which, in turn, generates charged particles. With regard to kerma these processes can under certain conditions be disregarded, or they can be accounted for by minor modifications or corrections. For electrons the situation is more difficult; they degrade all their energy either against binding energy or by the liberation of other electrons which can ionize in turn. The integral over the product of electron fluence times stopping power includes, therefore, part of the total energy repeatedly. It will accordingly exceed absorbed dose by a substantial factor. In calculations one can avoid this problem by using merely the 'primary' electron fluence spectrum, i.e. one can exclude the fluence of delta rays. In measurements this is generally not possible, because the delta rays fluence extends up to half of the maximum electron energy in the field and contributes a major part of the total fluence. A modified quantity is then required.

The modification is based on the same concept of excluding energy dissipation by part of the radiation field. But the category $K$ excludes here not a type of particles but merely electrons below a specified energy cut-off, $\Delta$. The somewhat different quantity that results will be called reduced cema; it involves the integral over the fluence of fast electrons with energies above $\Delta$ times their reduced stopping power. The reduced stopping power is the linear rate of energy loss of electrons that excludes the kinetic energy transmitted to 'fast' delta rays, i.e. delta rays with initial energy in excess of $\Delta$.

At first sight the concept of reduced cema appears fairly simple and consistent with the use of the conventional restricted LET (ICRU 1980, 1970). A closer consideration, however, shows that restricted LET is not applicable, because it excludes all collisions with energy loss of the electron in excess of $\Delta$, rather than excluding merely the kinetic energy of delta rays if it exceeds $\Delta$. At large cut-off values this makes little difference; at small cut-off values, however, it renders the present concept of restricted LET unsuitable. In fact restricted LET, in its present definition, vanishes for $\Delta=0$. One needs, therefore, a modified definition of a reduced $L E T$ that will, for the present discussion, be represented by the symbol $\Lambda_{\Delta}(T)$.

The reduced LET is the linear rate of energy loss of an electron minus the sum of the kinetic energies of fast delta rays released per unit track length. In the limit $\Delta=0$ this concept becomes equal to a quantity that has been utilized earlier by Spencer (1965), and also by Alm-Carlsson (1985), and that has been employed to give a formal definition of absorbed dose for an electron field:

$$
D=\frac{1}{\rho} \int_{0}^{\infty} \Lambda_{0}(T) \varphi_{\mathrm{e}}(T) \mathrm{d} T
$$


This equation is of conceptual importance but of no practical applicability, because the integral is predominantly determined by the fluence of delta rays of very low energy that can usually not be measured, and that is highly dependent on minor characteristics of the receptor geometry.

The reduced cema is different in character, because it depends only on the electron fluence above and at energy $\Delta$. Without analysing it in detail one can here state the equation that defines reduced cema in the continuous slowing down approximation (CSDA):

$$
C_{\Delta}=\frac{1}{\rho} \int_{\Delta}^{\infty} \Lambda_{\Delta}(T) \varphi_{\mathrm{e}}(T) \mathrm{d} T+\varphi_{\mathrm{e}}(\Delta) L(\Delta) \Delta / \rho
$$

$\varphi_{\mathrm{e}}(\Delta) L(\Delta) / \rho$ equals in the CSDA the number of electrons per unit mass that pass from kinetic energy in excess of $\Delta$ to energy below $\Delta$. The last term therefore represents the energy per unit mass of the track ends of all electrons with initial energy in excess of $\Delta$. It plays an important role also in cavity theory. In fact one finds that the concept of reduced cema is largely equivalent to notions used earlier in cavity theory (Spencer 1965).

\section{Conclusion}

Kerma and absorbed dose can be seen as special cases of a general concept that could be called energy conversion per unit mass, $C_{K}$, and that is defined in terms of energy converted from a specified category, $K$, of ionizing particles. If the category is all uncharged particles, one obtains, in essence, kerma. If it is all charged particles except delta rays, one obtains cema. If it is all ionizing particles except electrons below a specified energy $\Delta$, one obtains reduced cema. If $K$ is all ionizing particles, one obtains absorbed dose. The explicit definitions are not given here, but they are entirely analogous to the present ICRU definitions (ICRU 1980) that refer to the category of all ionizing particles.

The applicability of the new concept of cema with regard to charged particle fields, and specifically to space radiation, is evident. Reduced cema can play a useful role for calibration purposes in electron fields, and related concepts have, in fact, been employed widely in cavity theory.

To extend these considerations to the microdosimetric quantities, i.e. to stochastic variables, will be of similar interest, and it can be particularly helpful in clarifying the nature and applicability of computational approximations or of conditions of measurement.

\section{References}

Alm-Carlsson, (j., 1985, Theoretical basis for dosimetry. In The Dosimetry of Ionizing Radiation, edited by K. R. Kase et al. (London: Academic Press), vol. 2, pp. 1-75.

CASwell, R. S., and Coyne, J. J., 1972, Interaction of neutrons and secondary charged particles with tissue: secondary particle specctra. Radiation Research, 52, 448-470.

ICRU, 1970, Linear Energy Transfer, ICRU Report 16 (International Commission on Radiation Units and Measurements, Washington, DC).

ICRU, 1980, Radiation Quantities and Units, ICRU Report 33 (International Commission on Radiation Units and Measurements, Washington, DC).

ICRU, 1983, Microdosimetry, ICRU Report 36 (International Commission on Radiation Units and Measurements, Bethesda, Maryland).

ICRU, 1985, Determination of Dose Equivalents Resulting from External Radiation Sources, ICRU Report 39 (International Commission on Radiation Units and Measurements, Washington, DC). 
ICRU, 1988, Determination of Dose Equivalents from External Radiation Scurces, Part II, ICRU Report 43 (International Commission on Radiation Units and Measurements, Bethesda, Maryland).

Roesch, W. C., 1958, Dose for nonelectronic equilibrium conditions. Radiation Research, 9, 399-410.

SPENCER, L. V., 1965, Note on the theory of cavity ionization chambers. Radiation Research, $25,352-358$. 